\title{
Some applications of reliability based design optimization in engineering structures
}

\author{
C. López, A. Baldomir \& S. Hernández \\ Structural Mechanics Research Group, School of Civil Engineering, \\ University of A Coruña, Spain
}

\begin{abstract}
Structures are subject to uncertainty parameters that need to be considered in the design process. Reliability Based Design Optimization (RBDO) has become a powerful tool in achieving the optimum design when considering uncertainty data. In this work RBDO is applied in two different optimization approaches: size and topology optimization. There is a wide set of methods to solve RBDO problems, among them RIA (Reliability Index Approach), PMA (Performance Measure Approach) and SORA (Reliability Optimization and Reliability Assessment) are the most common in literature. For this work the SORA method has been chosen because it is easy to combine with commercial structural analysis and optimization codes due to the uncoupled nature of its formulation. It has been programmed in a MatLab code which manages the calls to structural analysis and optimization software needed to solve the RBDO problem. Finally, three application examples are used to compare the designs obtained with and without a probabilistic approach.

Keywords: $R B D O$, reliability index, size optimization, topology optimization, probability of failure.
\end{abstract}

\section{Introduction}

In engineering, some parameters used in the structural design process have an important random nature. Thus, load values might not be exactly known or material mechanical properties could not correspond to their theoretical values. Furthermore, as explained by Melchers [1], construction processes and product manufacturing have implicit inaccuracies and sources of error. From this fact, the idea is to provide a reliability approach to the structural analysis, in which some 
variables that traditionally had a fixed value now are considered as random variables. First contributions to this approach were made by Cornell [2], and since then important advances have been achieved in this field, as introduced in Haldar and Mahadevan [3] or Choi et al. [4], among others. Nowadays there are a large diversity of methods to perform reliability based structural analysis, and there are numerous research articles in this discipline, as Bandi et al. [5] or Chun et al. [6].

On the other hand, several structural analysis codes with optimization modules have been developed due to the growing importance of structural optimization in industry and the increasing computational facilities. Among the whole set of commercial packages, Abaqus [7], Optistruct [8], or Genesis [9] could be a representative sample of them. These codes are capable of analyzing and optimizing complex structural models with a large number of degrees of freedom in competitive computational times.

Thus, our focus is to use both capabilities: the power of commercial software and the efficiency of a decoupled RBDO method (SORA). Subsequently the code developed is this work is applied in examples with different approaches: size and topology optimization.

\section{Brief description of RBDO}

\subsection{General formulation of RBDO problems}

The most common mathematical formulation of RBDO problem is:

$$
\min F(\mathbf{x}, \mathbf{p})
$$

subject to:

$$
\begin{gathered}
g_{i}(\mathbf{x}, \mathbf{p}) \leq 0 ; \quad i=1, \ldots, n \\
P\left[G_{k}(\mathbf{x}, \mathbf{p}) \leq 0\right] \leq P_{f, k} \quad ; k=1, \ldots, m
\end{gathered}
$$

The objective is to minimize the function $F(\mathbf{x}, \mathbf{p})$, which depends on a combination of design variables $\mathbf{x}$ and fixed parameters $\mathbf{p}$. This objective function must satisfy a number of constraints $g_{i}(\mathbf{x}, \mathbf{p}) \leq 0$ related to the structural responses which depend, in the same way as the objective function, on vectors $\mathbf{x}$ and $\mathbf{p}$. The change in this optimization problem respect from the deterministic one is that the optimal structure must also satisfy a number of probabilistic constraints. These constraints consist of achieving the probability of failure of the structure, which must be lower than an imposed value $P_{f, k}$. Probability of failure is defined as the probability that the structure does not fulfil the required constraints at different limit-states of failure or service. Therefore, the probability of failure is always referred to the fulfillment of a limit-state function, which is expressed as $G_{k}(\mathbf{x}, \mathbf{p}) \geq 0$. For negative values of $G$, the structure is in a failure region and for positive values of $G$, the structure is in a safe region.

The probability of failure can be expressed as: 


$$
P_{f}=P[G(\mathbf{x}, \mathbf{p}) \leq 0]=\int \ldots \int_{G(\mathbf{x}, \mathbf{p}) \leq 0} H(\mathbf{x}, \mathbf{p}) d x d p
$$

where $H(\mathbf{x}, \mathbf{p})$ is the joint probability density function of $\mathbf{x}$ and $\mathbf{p}$. The analytical solution of this integral is usually quite complex, due to it is difficult to know the analytical expression of both $H(\mathbf{x}, \mathbf{p})$ and $G(\mathbf{x}, \mathbf{p})$. Approximation methods are normally used to evaluate (2) based on the performance function approximation (linear or higher order) and the use of first and second order moments of the random variables distributions. Assuming that the limit-state function is normally distributed, the probability of failure can be approximated as described by Cornell [2]:

$$
P_{f}=\Phi(-\beta)
$$

where $\phi$ and $\Phi$ are the standard normal probability density function and the standard normal cumulative distribution function, respectively. $\beta$ is known as reliability index.

Methods used to overcome the solution of problem (1) can be classified in three groups, according to Aoues and Chateauneuf [10]:

- Two level methods, which consider the probabilistic constraints in the optimization loop. This implies the need to solve nested loops, an outer loop for the optimization process and an inner loop for the reliability analysis.

- One level methods, which solve the problem in a single loop, avoiding reliability analysis.

- Uncoupled methods, which consist of separating the reliability analysis from the deterministic optimization problem. The problem become into a sequence of deterministic optimizations followed by the reliability analysis.

Below is brief description of the SORA method that will be used in this work.

\subsection{SORA (Sequential Optimization and Reliability Assessment)}

This method, proposed by Du and Chen [11], belongs to the uncoupled methods group. As discussed before, this family of methods avoids nested loops, making their solution faster in terms of computational time. In SORA method the reliability problem to be solved is expressed as follows:

$$
\min G_{k}\left(\mathbf{u}_{x}, \mathbf{u}_{p}\right)
$$

subject to:

$$
\beta=\beta_{\text {min }}
$$

Several algorithms has been proposed to solve the problem (4), and among them, one of the most popular is the AMV (Advanced Mean Value), described in $\mathrm{Wu}$ [12]. This algorithm works properly when the limit-state function is convex, but diverges or presents slow convergence when the function is concave. For such functions, the CMV algorithm (Conjugate Mean Value) has been developed, but is provides slow convergence when the function is convex. For this, Choi and Youn [13] propose a method named HMV (Hybrid Mean Value), which adaptively utilizes AMV and CMV methods for convex and concave limit state 


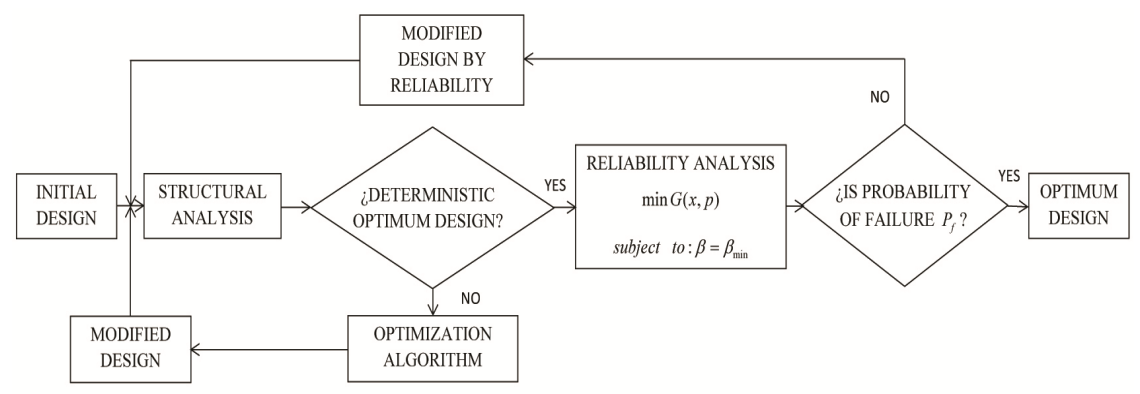

Figure 1: Flowchart of SORA method.

functions. This is the method chosen to accomplish the problem (4). A flowchart of the process is presented in Figure 1.

\section{Formulation and implementation of the problem}

The aim of this paper is to make a comparison of the results obtained in the deterministic and reliability based optimization for different structural configurations and different type of optimization: size and topology. The procedure followed in the three examples is the same. First of all, the deterministic optimization of the structural model is performed. This optimization is defined as minimizing the volume of the structure subject to the fulfillment of a number of stress constraints. This problem is expressed mathematically as:

$$
\min V
$$

subject to:

$$
|\sigma| \leq \sigma_{\text {máx }}
$$

Afterwards, the compliance of the optimal structure for each load case is obtained. This information will be used in the next step to formulate the RBDO problem. The objective is to obtain a structure of minimum volume which fulfills the stress conditions defined in (5.b), as well as a number of probabilistic constraints. In this case the limit state is defined by the stiffness (inverse of compliance) of the previous deterministic solution. The compliance $C_{i}$ of the structural design provided by the RBDO problem must have a lower value (hence, at least the same stiffness) in each load case than the value $C_{i}^{D E T}$ of the compliance of the structural design obtained in the deterministic optimization. For a structure with $j$ subcases, this problem is expressed:

$$
\min V
$$

subject to:

$$
\begin{gathered}
|\sigma| \leq \sigma_{\text {máx }} \\
P\left[C_{i}>C_{i}^{D E T}\right] \leq P_{f, i}
\end{gathered}
$$


To solve this problem, the SORA method has been implemented in a computational code programmed in MatLab [13], which allows getting the solution of large problems. The steps followed to obtain the problem solution are described below:

1. MatLab code calls Optistruct to obtain the optimal deterministic solution.

2. Then this code starts an iterative process executing the steps needed to solve the reliability problem through HMV method.

3. When this iterative process reaches convergence, the obtained point $\mathbf{u}^{k}$ is the Most Probable Failure Point (MPP), and thus the values of the random variables could be updated for the next deterministic optimization.

4. With these new updated values, the base code launches a new deterministic optimization process (solved with Optistruct) to obtain the new value of the optimal design variables.

5. Subsequently, HMV method is executed again to solve the reliability analysis problem. This leads to return again to step 2.

6. This sequence of steps is repeated until convergence.

Figure 2 shows a flowchart of the process described above. The central part of the chart shows the RBDO process from the MatLab code. In the right part of the chart it is shown the flowchart that follows Optistruct to solve the successive deterministic optimizations, while in the left part of the chart it is presented the flowchart of the HMV method implemented in the MatLab code as well.

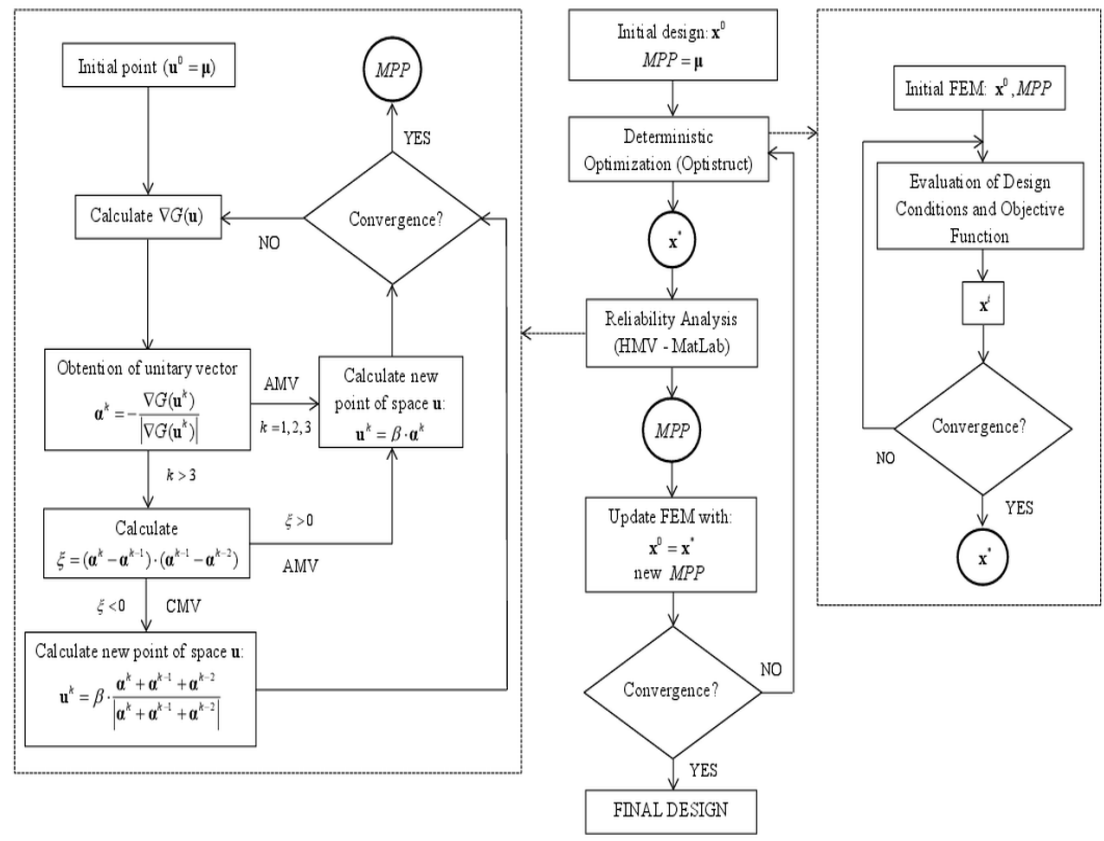

Figure 2: Flowchart SORA method, combining MatLab and Optistruct. 


\section{Application examples}

To demonstrate the efficiency of the developed code, several examples of different types of structural optimization have been proposed. All of them are solved using the procedure described in the previous section.

\subsection{Topology optimization: 2D bar truss structure}

The methodology explained in section 3 has been applied to a topology optimization example, defined in Baldomir [14]. The design variables of the problem are the relative density of material in all elements that define the design region. The initial problem is to obtain the optimal material distribution in the domain, taking into account the loads and boundary conditions specified. In particular, this is a 2D rectangular domain of $10 \mathrm{~m}$ length and $3 \mathrm{~m}$ height with two loads applied in point $\mathrm{A}$ as shown in Figure 3. This figure presents a scheme of the structural model, loads and boundary conditions, as shown in the supports placed in $\mathrm{B}$ and $\mathrm{C}$, respectively. Three load cases have been considered:

- Vertical load of $1000 \mathrm{kN}$.

- Horizontal load of $100 \mathrm{kN}$.

- Combination of two previous load cases.

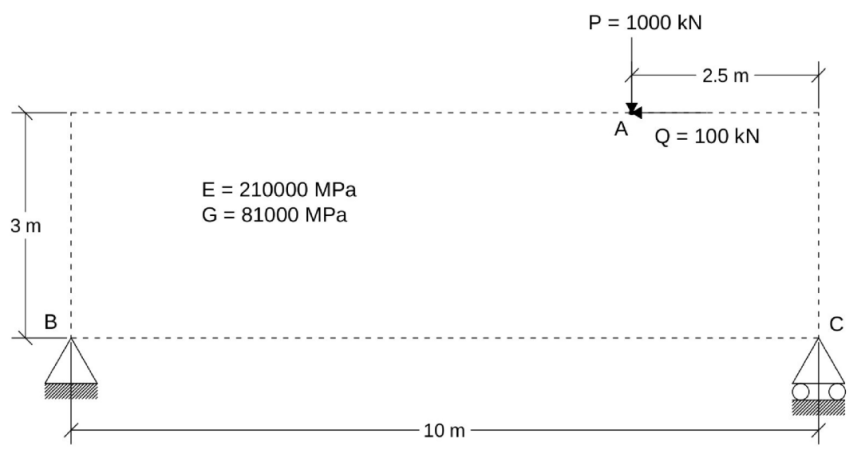

Figure 3: Finite element model of the rectangular domain

First, the deterministic topology optimization (DTO) is performed, and after that the reliability based topology optimization (RBTO) is accomplished so as to compare the results of both analyses. The DTO problem consists of minimizing the total volume of the structure subject to a number of stress constraints. In this example the Von Mises stress in all finite elements must not exceed $355 \mathrm{MPa}$. The problem is formulated mathematically as follows:

$\min V$

subject to

$$
|\sigma| \leq 355 \mathrm{MPa}
$$

After solving this problem in Optistruct, the compliance of the optimal structure for all load cases is: $C_{1}^{D E T}=9.374 \cdot 10^{6} \mathrm{~mm} / \mathrm{N}$, 
$C_{2}^{D E T}=1.872 \cdot 10^{5} \mathrm{~mm} / \mathrm{N}$ and $C_{3}^{D E T}=9.740 \cdot 10^{6} \mathrm{~mm} / \mathrm{N}$. The scheme obtained for the DTO is shown in Figure 4 and a conceptual 7-bar truss structure coherent with the solution.

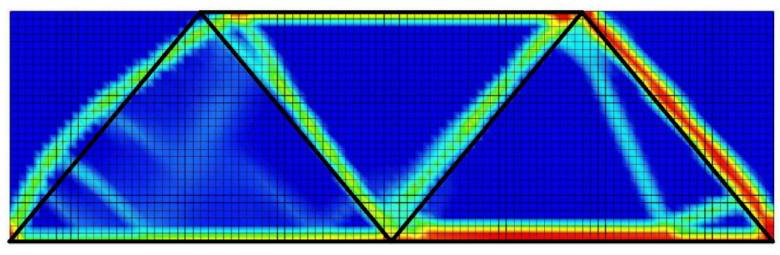

Figure 4: Conceptual design coherent with the DTO solution.

Afterwards, the RBTO is performed using the methodology exposed in section 3. The formulation of this problem can be expressed as:

$$
\min V
$$

subject to

$$
\begin{gathered}
|\sigma| \leq 355 \mathrm{MPa} \\
P\left[C_{i}>C_{i}^{D E T}\right] \leq P_{f, i}
\end{gathered}
$$

In this piece of research, two analyses have been performed with different probabilities of failure. For the first one, the probability of failure is $P_{f}=1.35 \cdot 10^{-3}$ and for the second is $P_{f}=2.86 \cdot 10^{-7}$, which correspond to reliability indexes $\beta=3$ and $\beta=5$, respectively.

Loads magnitudes and the Young's modulus of the material are the random variables, following both a normal distribution, with the mean and standard deviation values presented in Table 1.

Table 1: Mean and standard deviation of the random variables.

\begin{tabular}{lcccc}
\hline Random variable & Type of distribution & $\mu$ & $\sigma$ & $\delta$ \\
\hline Vertical load $(N)$ & Normal & $1.000 \cdot 10^{6}$ & $1.000 \cdot 10^{5}$ & 0.1 \\
Horizontal load $(N)$ & Normal & $1.000 \cdot 10^{5}$ & $1.000 \cdot 10^{4}$ & 0.1 \\
Young's modulus $(\mathrm{MPa})$ & Normal & $2.100 \cdot 10^{5}$ & $1.050 \cdot 10^{5}$ & 0.05 \\
\hline
\end{tabular}

The results obtained in the two RBTO analyses are shown in Figures 5 and 6, and a summary of both the DTO and RBTO results is shown in Table 2.

Table 2: $\quad$ Summary results of topology optimization.

\begin{tabular}{lccc}
\hline & Deterministic & \multicolumn{2}{c}{ Reliability based optimization } \\
& optimization & $\beta=3$ & $\beta=5$ \\
\hline Objective function $\left(\mathrm{mm}^{3}\right)$ & $7.372 \cdot 10^{7}$ & $1.111 \cdot 10^{8}$ & $1.669 \cdot 10^{8}$ \\
Initial fraction of volume $(\%)$ & 16.4 & 24.7 & 37.1 \\
\hline
\end{tabular}

It is remarkable that as the safety level increases, the number of elements in the mesh with high density levels increases. Besides, from a certain safety level, 
new ramifications interpreted as bars appear inside the domain. In this example, after solving the DTO it could be said that the optimal bar structure that arises from the solution is shown in Figure 4. On the other hand, after performing the RBTO with a safety level of $\beta=5$, the optimal structure arisen from the result is shown in Figure 6, where additional bars are generated in comparison with DTO.

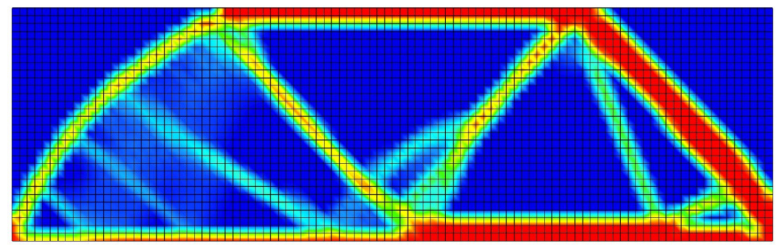

Figure 5: Results of the RBTO $(\beta=3)$.

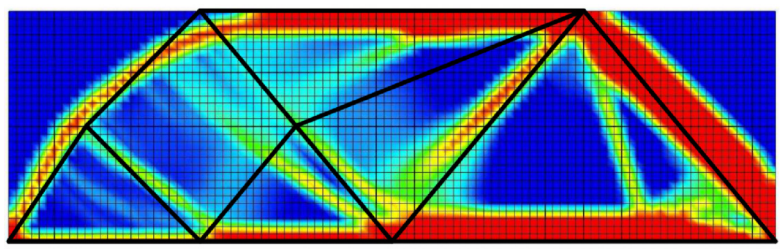

Figure 6: Conceptual design coherent with the RBTO with a safety level of $\beta=5$.

\subsection{Size optimization: 2D bar truss structure}

The objective of this section is to apply the methodology of section 3 to size optimization problems. This example is defined as a logical continuation of the design process accomplished in the previous section, where the topology optimization provides a structural scheme, but without a feasible sizing of the structure. In this section, the starting point is the layout of Figure 4, where a 7bar truss structure can be interpreted as presented in Figure 7.

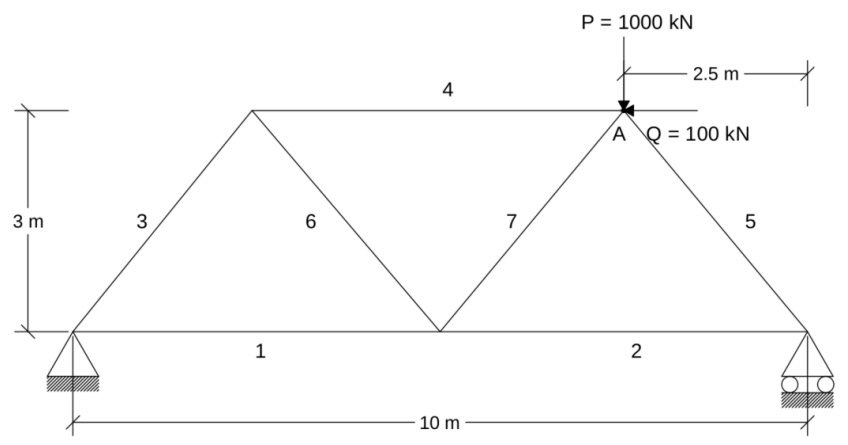

Figure 7: 7-bar truss structure for size optimization. 
The mechanical characteristics of the materials, the applied loads and load cases considered in the new structure are the same as in the previous example. The design variables are the cross-sectional areas in all bars of the truss structure. The deterministic problem is defined by minimizing the total volume of the structure subject to stress constraints. The constraints to be fulfilled are that the axial stresses in all bars must be lower than $355 \mathrm{MPa}$. The mathematical formulation of this problem can be written as:

$$
\min V
$$

subject to:

$$
\left|\sigma_{i}\right| \leq 355 M P a ; i=1, \ldots, 7
$$

After solving this problem, the compliances of the optimal structure for all load cases are obtained: $C_{1}^{D E T}=1.119 \cdot 10^{7} \mathrm{~mm} / \mathrm{N}, C_{2}^{D E T}=1.871 \cdot 10^{5} \mathrm{~mm} / \mathrm{N}$ and $C_{3}^{D E T}=1.134 \cdot 10^{7} \mathrm{~mm} / \mathrm{N}$. These values are used to impose the probabilistic constraints as stated in section 3 .

As in the previous example, two analyses have been carried out imposing probabilities of failure of $P_{f}=1.35 \cdot 10^{-3}$ and $P_{f}=2.86 \cdot 10^{-7}$, which correspond to reliability indexes $\beta=3$ and $\beta=5$, respectively. The random variables are the loads values and the Young's modulus of the material, whose uncertainty data is shown in Table 1.

Moreover, an additional analysis has been performed. Considering the layout of Figure 6, a new 13-bar truss structure can be defined as shown in Figure 8.

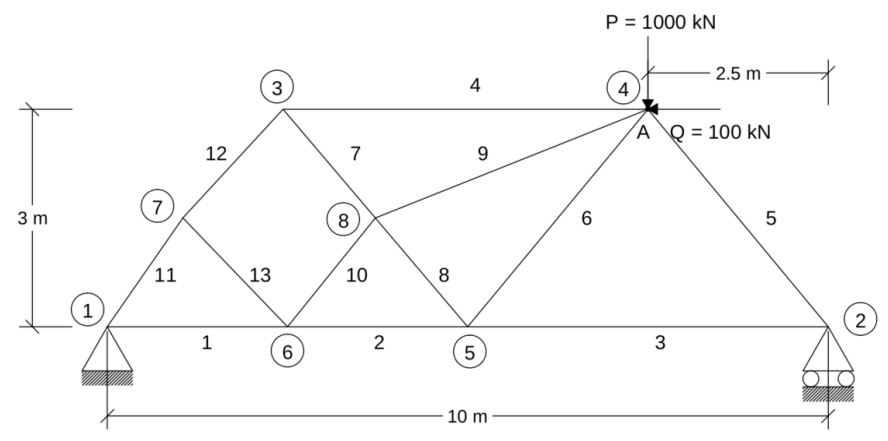

Figure 8: 13-bar truss structure for size optimization.

The analysis performed consists of solving the RBDO problem defined above for this 13-bar structure imposing a safety level of $\beta=5$ for the probabilistic constraint (6.c). The compliances imposed in this condition are taken from the 7bar example. This assures that the 13-bar structure obtained will be as rigid as the 7-bar one. Node coordinates that define the truss structure are shown in Table 3. 
Table 3: Node coordinates of the 13-bar structure.

\begin{tabular}{lcccccccc}
\hline NODES & 1 & 2 & 3 & 4 & 5 & 6 & 7 & 8 \\
\hline Horizontal-coordinate $(m)$ & 0 & 10 & 2.5 & 7.5 & 5 & 2.5 & 1.05 & 3.75 \\
Vertical-coordinate $(m)$ & 0 & 0 & 3 & 3 & 0 & 0 & 1.5 & 1.5 \\
\hline
\end{tabular}

Table 4 presents a summary of the results for the deterministic and the RBDO approaches for the 7-bar structure, as well as the results of the RBDO carried out for the 13-bar structure.

Table 4: Summary results of size optimization.

\begin{tabular}{lcccc}
\hline & $\begin{array}{c}\text { DO 7-bar } \\
\text { structure }\end{array}$ & \multicolumn{2}{c}{ RBDO 7-bar structure } & $\begin{array}{c}\text { RBDO 13- } \\
\text { bar structure } \\
\beta=5\end{array}$ \\
\hline Obj. Function $\left(\mathrm{mm}^{3}\right)$ & $4.112 \cdot 10^{7}$ & $7.195 \cdot 10^{7}$ & $9.813 \cdot 10^{7}$ & $9.704 \cdot 10^{7}$ \\
\hline
\end{tabular}

For the 7-bar structure, it can be outlined that as the safety level increases, the cross-sectional areas increase, causing a weight increment in the structure. Comparing both structures for a safety level of $\beta=5$, it is noticeable that the 13-bar structure provides a lower volume than the 7-bar structure, which agrees with the results obtained in the RBTO approach performed in section 4.1. In this section, for a safety level of $\beta=5$ the optimal layout of Figure 6 leads to a 13bar structure instead of a 7-bar structure.

\subsection{D Topology optimization: fuselage aircraft}

The methodology described in section 3 was also applied in a topology example of a simplified 3D fuselage aircraft. A side view of the finite element model representing the design region is shown in Figure 9. The model has $10 \mathrm{~m}$ length and the radio goes from $2.1 \mathrm{~m}$ to $0.45 \mathrm{~m}$. All movements are constrained in the front part of fuselage and two set of loads are applied in the rear part: those transmitted by the vertical tail plane to the fuselage and the loads corresponding to the rear fuselage tail cone.

The results obtained are shown in Figures 10 and 11 and the numerical values of optimum volume are summarized in Table 5.

It can be observed an important increase in volume $(17.4 \%)$ when a reliability approach is performed. As expected an increase in safety level leads to an increase in material volume. However the volume increase is not only produced by a member size increase but also by a change in the structural layout.

Table 5: Summary of topology optimization results.

\begin{tabular}{lcc}
\hline & $\begin{array}{c}\text { Deterministic } \\
\text { optimization }\end{array}$ & $\begin{array}{c}\text { Reliability based } \\
\text { optimization }(\beta=3)\end{array}$ \\
\hline Objective function $\left(\mathrm{mm}^{3}\right)$ & $3.495 \cdot 10^{9}$ & $4.111 \cdot 10^{9}$ \\
Initial fraction of volume (\%) & 3.58 & 4.31 \\
\hline
\end{tabular}




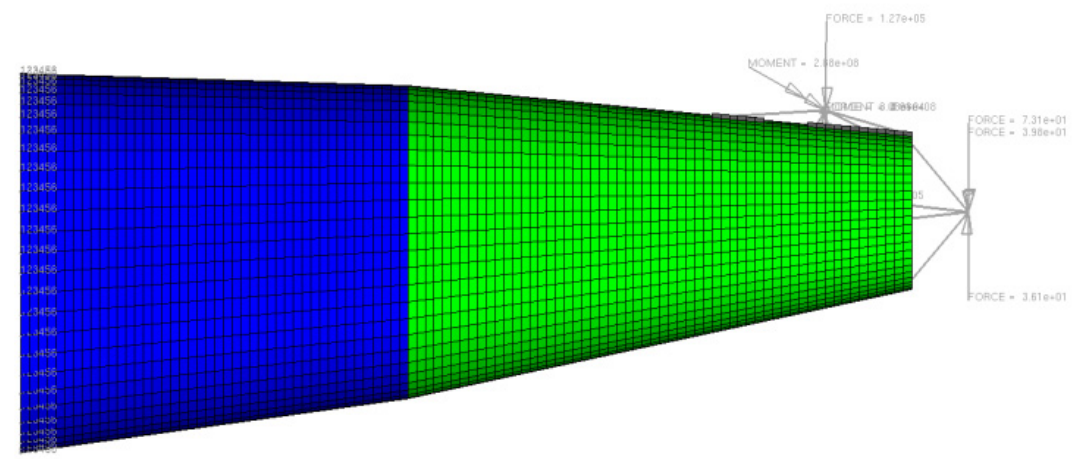

Figure 9: Finite element model.
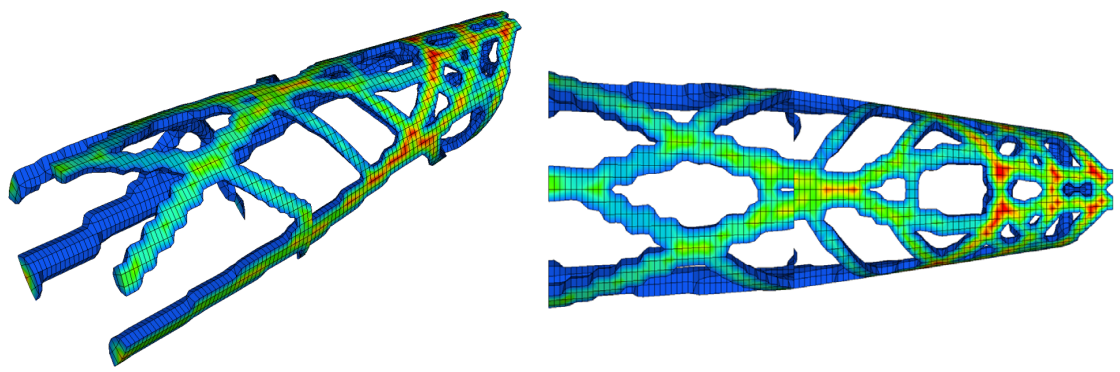

Figure 10: Deterministic topology optimization.
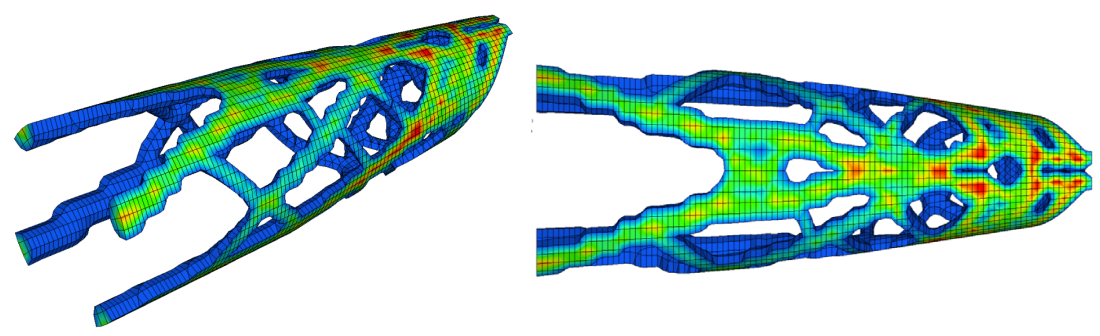

Figure 11: $\operatorname{RBTO}$ solution $(\beta=3)$.

\section{Conclusions}

In this paper is proposed a strategy to combine commercial software with an efficient RBDO method through a Matlab code. It was stated the efficiency of this strategy to solve RBDO problems with a large number of design variables and several state limits.

It can also be concluded that the higher reliability requested in every structural design, the heavier the optimal structural designs are. In topology 
optimization, when requesting a high reliability, can produce a change in structural layout, not only an increasing in the member size. This involves the development of new structural schemes.

It was also demonstrated that the size RBDO result from a previous RBTO layout provide better results than those from a DTO layout.

\section{References}

[1] Melchers, R.E., Structural Reliability Analysis and Prediction, John Wiley \& Sons, Chichester, England.

[2] Cornell, C.A., A probability-based structural code. Probabilistic Design of Reinforced Concrete Buildings, Americal Concrete Institute 1969; 111146.

[3] Haldar, A. and Mahadevan, S., Probability, reliability, and statistical methods in engineering design. John Wiley \& Sons, New York. 2000.

[4] Choi, So. Grandhi, R. and Canfield, R., Reliability-based structural design. Springer-Verlag, London. 2007.

[5] Bandi, P., Tovar, A. and Renaud, J.E. Reliability based designs for crashworthiness: Decision under uncertainty/uncertainty modeling. SAE Technical Paper 2010-01-0909, 2010.

[6] Chun, J., Song, J. and Paulinho, G.H. System reliability based topology optimization of structures under stochastic excitations. Safety, Reliability, Risk and Life-Cycle Performance of Structures and Infrastructures, ICOSSAR 2013. 2013; 4935-4942.

[7] Abaqus 6.12-1, Dassault Systèmes Simulia Corp., RI, USA, 2012.

[8] Altair Optistruct User Manual. Version 12, Altair Engineering Inc. 2013.

[9] Genesis 12.2. Vanderplaats R\&D, Inc. 2012.

[10] Aoues, Y. and Chateauneuf, A. Benchmark study of numerical methods for reliability based design optimization. SMO 2010; 41: 277-294.

[11] Du, X. and Chen, W., Sequential Optimization and Reliability Assessment Method for Efficient Probabilistic Design. Journal of Mechanical Design 2004; 126: 225-233.

[12] Wu, Y.T., 1994, Computational Methods for Efficient Structural Reliability and Reliability Sensitivity Analysis AIAA Journal, Vol. 32, No's, 1717-1723.

[13] Choi, K.K. and Youn, B.D. Hybrid Analysis Method for Reliability-Based Design Optimization, ASME 2001, Pittsburgh, Pennsylvania, September 9-12.

[14] Baldomir, A. Análisis y optimización en entornos de incertidumbre de estructuras de ingeniería civil y aeronáutica. Tesis Doctoral Universidade da Coruña, 2010; 38-42. 